\title{
LHomme
}

L'HOMME Revue française d'anthropologie

$183 \mid 2007$

Comment être parents ?

\section{Gabriele vom Bruck \& Barbara Bodenhorn, eds, An Anthropology of Names and Naming}

Cambridge-New York, Cambridge University Press, 2006, 290 p., bibl., index

\section{Charles Macdonald}

\section{(2) OpenEdition}

\section{Journals}

Édition électronique

URL : http://journals.openedition.org//homme/9741

DOI : 10.4000/lhomme.9741

ISSN : 1953-8103

Éditeur

Éditions de l'EHESS

\section{Édition imprimée}

Date de publication : 1 septembre 2007

Pagination : 219-220

ISSN : 0439-4216

Référence électronique

Charles Macdonald, « Gabriele vom Bruck \& Barbara Bodenhorn, eds, An Anthropology of Names and Naming », L'Homme [En ligne], 183 | 2007, mis en ligne le 28 juin 2007, consulté le 21 septembre 2020 URL : http://journals.openedition.org//homme/9741 ; DOI : https://doi.org/10.4000/lhomme.9741

Ce document a été généré automatiquement le 21 septembre 2020

〔c École des hautes études en sciences sociales 


\section{Gabriele vom Bruck \& Barbara Bodenhorn, eds, An Anthropology of Names and Naming}

Cambridge-New York, Cambridge University Press, 2006, 290 p., bibl., index

\section{Charles Macdonald}

1 L'ANTHROPOLOGIE des noms personnels ou ethno-anthroponymie commence à faire recette. On trouve ainsi sous le titre ambitieux Une anthropologie [sociale ou culturelle ?] des noms [personnels ?] et des pratiques de nomination une collection d'articles présentés lors d'un colloque organisé à Cambridge en 1999.

Ce volume comprend une assez longue introduction et dix articles traitant des noms personnels aux États-Unis (Linda Layne), en Nouvelle-Guinée (André Itéanu), en Amazonie (Stephen Hugh-Jones), à Madagascar (Maurice Bloch, Michael Lambek), chez les Iñupiaq d'Alaska (Barbara Bodenhorn), en Mongolie (Caroline Humphrey), chez les esclaves africains en Afrique et aux États-Unis (Susan Benson), et au Yémen (Gabriele vom Bruck). Un des dix articles traite des toponymes en Afrique du Sud (Thomas Blom Hansen).

3 La loi du genre oblige les coordinateurs d'un tel ouvrage à conférer, dans une introduction, une unité à des propos hétéroclites. La tentative ne réussit pas toujours, ce qui explique peut-être que chaque article est aussi précédé d'une notice explicative, comme dans les catalogues d'art.

Dans leur préface, Gabriele vom Bruck et Barbara Bodenhorn annoncent qu'elles n'ont "trouvé dans le champ de l'analyse anthropologique récente rien qui rassemble l'ethnographie comparative et une théorie générale des processus [de nomination] " (p. XII). C'est qu'elles ont mal cherché. L'ouvrage publié par Josiane Massard-Vincent et Simonne Pauwels en $1999^{1}$, qui n'est pas cité, leur aurait fourni au moins quelques éléments dans ce sens. 
5 J'y esquissais en effet les grandes lignes d'une méthodologie comparative. Le titre de leur introduction comporte le mot entangled (« enchevêtré, emmêlé ») et se réfère à un concept de psychologie phénoménologique que j'avais signalé, dans un autre article, à propos des noms personnels ${ }^{2}$. Ailleurs dans le livre des références essentielles manquent. C'est le cas, par exemple, de l'article de Barbara Bodenhorn sur les Iñupiaq qui ne mentionne ni les articles pertinents de Bernard Saladin d'Anglure ${ }^{3}$, ni ceux de Pierre Robbe ${ }^{4}$ ou de Robert Gessain ${ }^{5}$, consacrés à des groupes inuit du Centre et de l'Est (Groenland) certes, mais incontestablement partie prenante du même ensemble culturel en ce qui concerne les noms personnels.

6 L'introduction recense les éternelles références en philosophie linguistique (Mill, Kripke, Searle, Wittgenstein, etc.) et puise abondamment dans la "French theory " (Derrida, Bourdieu, Foucault, Lacan, Althusser...). Se promenant dans le pré de l'ethnographie, les auteurs composent quelques bouquets de ressemblances et de différences, en examinent quelques aspects et en offrent des interprétations à la croisée de multiples disciplines (psychologie, ethnologie, philosophie, sémiologie, pragmatique). Par exemple, page 18, on se demande si "partager un nom rend la frontière entre individus floue ». Page 19, on lit : « S'attribuer un nom à soi-même, dans un acte d'illocution ritualisé, constitue d'abord un acte d'auto-objectivation qui élimine la dichotomie entre signifiant et signifié ». Le mot "nécronyme », ici comme ailleurs dans le volume, n'est pas utilisé dans son sens correct (celui qu'utilisaient Rodney Needham et Claude Lévi-Strauss qui l'ont mis en circulation). Les auteurs, comme une majorité d'anthropologues, sont attirés par le problème de la relation entre le nom et la personne et les aspects illocutoires et déictiques connexes. Ils ne font pas la différence entre les deux niveaux du phénomène, celui des lexèmes et celui des catégories onomastiques. Ils ont ainsi du mal à passer d'une sémiologie à une sociologie des noms.

On trouvera de bons, voire d'excellents articles, notamment sur les Orokaiva de Nouvelle-Guinée, sur les noms mongols, sur les noms d'esclaves. De timides tentatives comparatistes sont esquissées pour les noms amazoniens et africains.

8 L'amateur de curiosités onomastiques et de détails ethnographiques trouvera dans cet ouvrage des données assez détaillées et précisément analysées au cas par cas, et d'un grand intérêt par elles-mêmes. Le théoricien ne trouvera pas grand-chose de nouveau ou de solide pour remplir la promesse du titre, celle d'une théorie anthropologique des noms personnels qui soit réellement comparative. Le concept même de "nom » reste vaguement défini et il est utilisé au fil de l'ouvrage dans plusieurs acceptions différentes mais non distinguées.

\section{NOTES}

1. Josiane Massard-Vincent \& Simonne Pauwels, eds, D'un nom à l'autre en Asie du SudEst : approches ethnologiques, Paris, Karthala, 1999.

2. Cf. Charles Macdonald, «Can Personal Names be Translated? ", IIAS Newsletter, 2005, $36: 14$. 
3. Voir en particulier, « Nom et parenté chez les Esquimaux Tarramiut du NouveauQuébec (Canada) », in Jean Pouillon \& Pierre Maranda, eds, Échanges et Communications : mélanges offerts à Claude Lévi-Strauss à l'occasion de son $60^{\circ}$ anniversaire, Paris-La Haye, Mouton, 1970 : 1013-1039.

4. Pierre Robbe, « Les noms de personne chez les Ammassalimiut », Études Inuit/Inuit Studies, 1981, 5 (1) : 45-82.

5. Robert Gessain, « Nom et réincarnation chez les Ammassalimiut », Boréales, 1980, 5 (15-16) : 407-419.

\section{AUTEUR}

\section{CHARLES MACDONALD}

CNRS, Faculté de médecine, Unité d'anthropologie, Marseille. c.macdonald@wanadoo.fr 